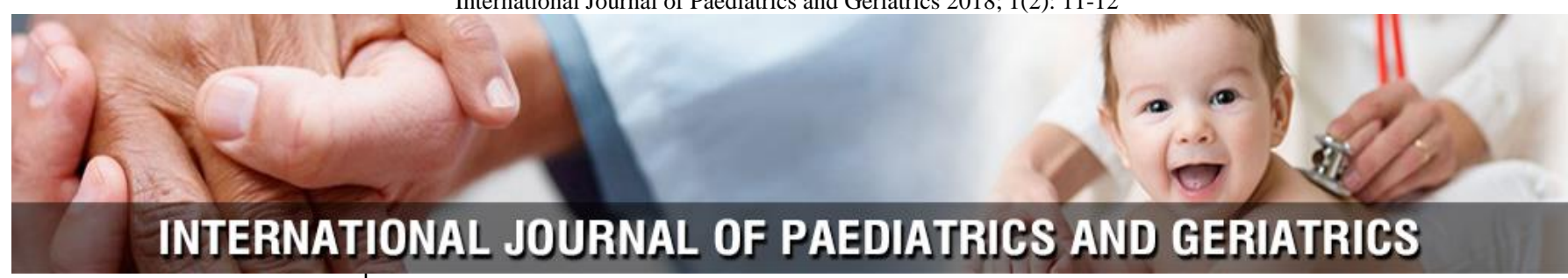

P-ISSN: 2664-3685

E-ISSN: $2664-3693$

www.paediatricjournal.com IJPG 2018; 1(2): 11-12

Received: 18-05-2018

Accepted: 21-06-2018

Dr. Shivjot Sharma

Department of Paediatrics, Punjab Institute of Medical Sciences, Punjab, India

\section{Evaluation of type of headache in children}

\section{Dr. Shivjot Sharma}

DOI: $\underline{\text { https://doi.org/10.33545/26643685.2018.v1.i2a.13 }}$

\begin{abstract}
Background: Headache is a major health concern not only because it is a disabling disease. The present study was conducted to evaluate the types of headaches in children.

Materials \& Methods: This study was conducted on 1530 children of both gender. A self-responding questionnaire was given to all patients to fill it. A thorough clinical examination was performed. Factors such as duration of headache, type of headache and family history of headache was also recorded.

Results: Out of 1530, 680 (44.5\%) had headache. Migraine was seen in 36\%, unspecified headache in $10 \%$, tension type was seen in $24 \%$, trigeminal autonomic cephalgia in $15 \%$ and primary stabbing headache in 5\%. The difference was significant $(P<0.05)$. Father had $12 \%$, mother had $7 \%$, both had $4 \%$ and siblings had $2 \%$ of family history of headache. The difference was significant $(\mathrm{P}<0.05)$.

Conclusion: Migraine in children is among various types of headaches. The increase in number of cases day by day demands assessment of all possible parameters.
\end{abstract}

Keywords: Children, headache, migraine

\section{Introduction}

Headache is a common complaint in children. Headache is a major health concern not only because it is a disabling disease, but also because of high utilization of health care system and due to work absenteeism ${ }^{[1]}$. World Health Organization has given the disability score of 0.7 to migraine and according to them it is one of the most debilitating illnesses. Moreover, adolescents with headache prefer taking analgesics themselves over visiting a physician and this may lead to development of medication overuse headache which is refractory to most of the available treatments ${ }^{[2]}$.

In school children the most common form are primary headaches, defined as headaches not associated with an underlying disorder (group 1-4, IHS classification ${ }^{[3]}$. The median frequency of headaches (1-month to lifetime prevalence) in 50 population-based studies was $58.4 \%$ in school children, while the prevalence of migraine headaches was $7.7 \%$. The ratio of girls to boys was 1.5:1 (all headaches) and 1.7:1 (migraines). Secondary headaches (headaches associated with an underlying disease) are uncommon in patients with recurrent headaches. The prevalence of headaches in school children (11-18 years of age) in Turkey was $34.1 \%$ for primary headaches and $4.4 \%$ for secondary. The most common causes of secondary headaches were viral respiratory infections (29-39\%) and mild skull injuries ${ }^{[4]}$. The present study was conducted to evaluate the types of headaches in children.

\section{Materials \& Methods}

This study was conducted in department of Pediatrics. It included 1530 children of both gender. All were informed regarding the study and written consent was obtained. Ethical approval was obtained prior to the commencement of the study.

Information such as name, age, gender etc was recorded. A self-responding questionnaire was given to all patients to fill it. A thorough clinical examination was performed. Factors such as duration of headache, type of headache and family history of headache was also recorded. Results thus obtained were subjected to statistical analysis. $\mathrm{P}$ value $<0.05$ was considered significant.

\section{Results}

Table I: Children with headache

\begin{tabular}{|c|c|c|}
\hline Total & Headache & Percentage \\
\hline 1530 & 680 & $44.5 \%$ \\
\hline
\end{tabular}

ding Auth

Department of Paediatrics, Punjab Institute of Medical Sciences, Punjab, India 
Table I shows that out of $1530,680(44.5 \%)$ had headache.

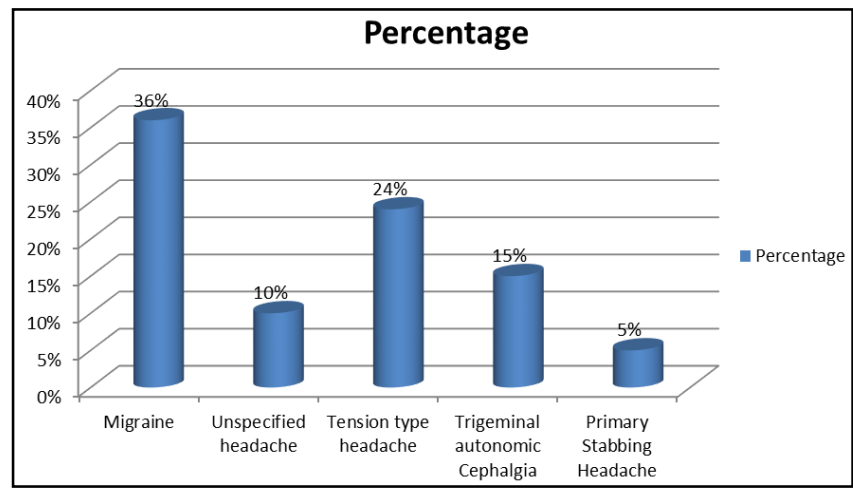

Graph 1: Type of headache

Graph I shows that migraine was seen in $36 \%$, unspecified headache in $10 \%$, tension type was seen in $24 \%$, trigeminal autonomic cephalgia in $15 \%$ and primary stabbing headache in $5 \%$. The difference was significant $(\mathrm{P}<0.05)$.

Table 2: Family history

\begin{tabular}{|c|c|c|}
\hline Family members & Percentage & \multirow{2}{*}{ P value } \\
\hline Father & $12 \%$ & \multirow{2}{*}{0.04} \\
\hline Mother & $7 \%$ & \\
\hline Both & $4 \%$ & \\
\hline Siblings & $2 \%$ & \\
\hline
\end{tabular}

Table II shows that father had $12 \%$, mother had $7 \%$, both had $4 \%$ and siblings had $2 \%$ of family history of headache. The difference was significant $(\mathrm{P}<0.05)$.

\section{Discussion}

Previous studies differ regarding the overall prevalence of primary headaches in children and also regarding the type of primary headaches. While some studies had described migraine as the most prevalent headache in adolescents, other studies have found tension type headache to be the most common type. A recent review suggested that despite high prevalence of headache throughout the world, most of the epidemiological studies were conducted in Western Europe and North America ${ }^{[5-6]}$. The present study was conducted to evaluate the types of headaches in adolescents. In this study, we found that out of 1530, 680 (44.5\%) had headache. Straube et al. ${ }^{[7]}$ searched the PubMed database for pertinent publications that contained the terms "primary headache and children/adolescent and risk factors/prevalence." Articles published in either English or German up to April 2013 were considered. Headaches are becoming more common among school children. At present, $66 \%$ to $71 \%$ of 12 - to 15 - year-olds have at least one headache every three months, and $33 \%$ to $40 \%$ have at least one per week. Headache is often accompanied by other physical and/or emotional manifestations. Studies from Scandinavia reveal increasing prevalence in age groups from 8 years of age and upward. Various studies have identified the following risk factors for headache or for its cornification, a dysfunctional family situation, the regular consumption of alcohol, caffeine ingestion, smoking, a low level of physical activity, physical or emotional abuse, bullying by peers, unfair treatment in school, and insufficient leisure time.

We observed that migraine was seen in $36 \%$, unspecified headache in $10 \%$, tension type was seen in $24 \%$, trigeminal autonomic cephalgia in $15 \%$ and primary stabbing headache in $5 \%$. Father had $12 \%$, mother had $7 \%$, both had $4 \%$ and siblings had $2 \%$ of family history of headache.

Primary headaches are benign, recurrent headaches not caused by underlying disease or structural problems. For example, migraine is a type of primary headache. While primary headaches may cause significant daily pain and disability, they are not dangerous. Secondary headaches are caused by an underlying disease, like an infection, head injury, vascular disorders, brain bleed or tumors. Secondary headaches can be harmless or dangerous. Certain "red flags" or warning signs indicate a secondary headache may be dangerous ${ }^{[8]}$.

Milde et al. [9] analyzed 50 population-based studies reporting the prevalence of headache and / or migraine in children and adolescents $(<20 y)$. The estimated prevalence of headache over periods between 1 month and lifetime in children and adolescents was $58.4 \%$. Females are more likely to have headache than males. The prevalence of migraine over periods between 6 months and lifetime is $7.7 \%$. Females are more likely than males to have migraine. Regional differences in prevalence of migraine, though statistically significant, may not be of clinical significance. The change in the IHS's criteria for the diagnosis of migraine was not associated with any significant change in the prevalence of migraine.

\section{Conclusion}

Migraine in children is among various types of headaches. The increase in number of cases day by day demands assessment of all possible parameters.

\section{References}

1. Guidetti V, Galli F. Evolution of headache in childhood and adolescence: an 8-year follow-up. Cephalalgia 1998; 18:449-454.

2. Aromaa M, Rautava P, Helenius H, Sillanpaa M. Factors of early life as predictors of headache in children at schoolventry. Headache. 1998; 38:23-30.

3. Ozge A, Bugdayci R, Sasmaz T et al. The sensitivity and specificity of the case definition criteria in diagnosis of headache: a schoolbased epidemiological study of 5562 children in Mersin. Cephalalgia. 2002; 22:791-798.

4. Shivpuri D, Rajesh MS, Jain D. Prevalence and characteristics of migraine among adolescents: A questionnaire based study. Indian Pediatrics. 2003; 40:665-669.

5. Laurell K, Larsson B, Eeg-Olofsson O. Headache in schoolchildren: agreement between different sources of information. Cephalalgia. 2003; 23:420-428.

6. Dooley JM, Gordon KE: Headaches in childhood. Can J Neurol Sci. 2004; 31:291-2.

7. Straube A, Heinen F, Ebinger F, Von Kries R. Headache in school children: prevalence and risk factors. Dtsch Arztebl Int. 2013; 110(48):811-18.

8. Hagen K, Thoresen K, Stovner LJ, Zwart JA. High dietary caffeine consumption is associated with a modest increase in headache prevalence: results from the Head-HUNT Study. J Headache Pain 2009; 10:1539.

9. Milde-Busch A, Blaschek A, Borggräfe I, Heinen F, Straube A, von Kries R. Associations of diet and lifestyle with headache in high-school students: results from a cross-sectional study. Headache. 2010; 50:1104-14. 